\title{
MORTE ENCEFÁLICA E DOAÇÃO DE ÓRGÃOS E TECIDOS: CONHECENDO A TAXA DE DOAÇÕES EFETIVAS EM UM HOSPITAL DA SERRA GAÚCHA
}

\author{
Encephalic death and organ and tissue donation: \\ knowing the rate of effective donations at a hospital in Serra Gaúcha
}

\author{
Marina Brandalise ${ }^{1}$, Rosana Pinheiro Lunelli ${ }^{1}$, Ruy de Almeida Barcellos², Patricia Kelly Wilmsem Dalla Santa Spada ${ }^{1}$, \\ Hugo Diogo Folchini de Castilhos ${ }^{3}$
}

\begin{abstract}
RESUMO
Há significativa discrepância entre a demanda de pacientes que aguardam nas filas de transplantes quando comparada ao número de transplantes efetivados. Objetivo: mensurar a taxa de doações de órgãos e tecidos em pacientes com diagnóstico de morte encefálica no período de 2013 a 2017. Método: estudo transversal desenvolvido na cidade de Caxias do Sul/RS. A coleta de dados foi realizada no primeiro semestre de 2018 e os dados foram analisados descritivamente por frequências absolutas e relativas. Calculou-se o odds ratio e associações pelo teste Qui-Quadrado de Wald, considerando-se $p<0,05$ como significância estatística. Resultados: A taxa de doação de órgãos e tecidos foi de $48,15 \%$ em uma população de 216 pacientes, e a negativa familiar se destacou como principal motivo para a não doação $(28,24 \%)$. Dentre as associações que se correlacionam com o desfecho da doação destacam-se o estado civil e AVC como causa da ME. Conclusão: Novos estudos com desenhos mais robustos em diferentes centros e com populações maiores são necessários, para que se possa avaliar de forma mais profunda as associações e desfechos. São necessárias ações de sensibilização e conscientização referentes à negativa familiar como causa para a não doação.
\end{abstract}

Descritores: Morte Encefálica; Transplante de Órgãos; Obtenção de Tecidos e Órgãos; Enfermagem.

\footnotetext{
Instituições:

${ }^{1}$ Centro Universitário da Serra Gaúcha - FSG, Caxias do Sul/RS

2 Hospital de Clínicas de Porto Alegre - HCPA, Porto Alegre/RS

${ }^{3}$ Hospital Nossa Senhora de Pompéia - Caxias do Sul/RS
}

Correspondência:

Marina Brandalise

Rua das Araucárias, 155 - Apto. 306, CEP 95270-000, Flores da Cunha/RS

Tel.: (54) 98118-9846

E-mail: nina.brandalise@hotmail.com

Recebido em: 24/06/2018

Aceito em: 16/07/2018

\section{INTRODUÇÃO}

As funções pulmonares e cardíacas são imprescindíveis para sustentação da vida humana; todavia, é possível que haja manutenção das funções vitais por meio de aparelhos, mesmo quando não há funcionamento do encéfalo, procedimento este também conhecido como "coma depassé", que, historicamente, sucedeu o parecer de morte encefálica (ME) desenvolvido por um grupo de neurologistas franceses, em 1959. ${ }^{1}$

As causas mais frequentes da ME são oriundas de traumatismo cranioencefálico (TCE) e acidente vascular encefálico (AVE), somando mais de $90 \%$ no total dos potenciais doadores; porém, outras causas de menor prevalência são apontadas, incluindo infecções do sistema nervoso central, tumores cerebrais e anóxia pós-parada cardiorrespiratória $(\mathrm{PCR})^{2,3}$ Através de critérios de avaliação, a morte encefálica passou a 
ter importante papel no diagnóstico diferencial dos pacientes, justamente por estar atrelada à doação de órgãos e tecidos. ${ }^{4}$

Para pacientes portadores de alguma insuficiência funcional de órgãos essenciais, o transplante de órgãos é, na maioria das vezes, a única alternativa terapêutica. Não somente no Brasil, mas em outros países, observase uma significativa discrepância na demanda de pacientes que aguardam nas filas de transplante quando comparada ao número de transplantes efetivados. ${ }^{5}$ Entre o período de janeiro a junho de 2016, o número de potenciais doadores foi de 4.970 pacientes, mas apenas 1.434 foram doadores efetivos, uma vez que no Rio Grande do Sul apenas 284 pacientes foram doadores efetivos. ${ }^{6}$ Já em 2017, no mesmo período analisado, obtiveram-se 5.309 potenciais doadores e somente 1.666 tornaram-se doadores efetivos. ${ }^{7}$

Dentre as causas da não concretização da doação de órgãos e tecidos, pode-se citar parada cardíaca, não reconhecimento do potencial doador pela equipe de saúde, contraindicações clínicas, crenças religiosas e negativa familiar. ${ }^{8}$ Nos motivos mais frequentes para a negativa familiar, destacam-se a oposição do próprio paciente quando vivo à doação, apego familiar ao corpo, não aceitação ou compreensão do processo de ME, ansiedade em relação à morte, incapacidade à tomada de decisões frente à notícia da perda do ente querido, receio de mutilação do corpo, crenças populares e religiosas, bem como insensibilidade dos profissionais de saúde frente à abordagem familiar. ${ }^{9}$

Em virtude desse tema propagar interesse mundial por exercer grande impacto na saúde coletiva, a execução desta pesquisa justifica-se pela possibilidade de apontar fatores que auxiliem possíveis melhorias na assistência prestada aos pacientes com diagnóstico de ME e seus familiares, visando o aumento das doações. Desta forma, o objetivo da pesquisa é mensurar a taxa de doações de órgãos e tecidos em pacientes com diagnóstico de morte encefálica, correlacionando variáveis do processo de doação ao desfecho doação efetiva no período de 2013 a 2017.

\section{MÉTODO}

Trata-se de estudo retrospectivo e transversal, desenvolvido pela Comissão Intra-Hospitalar de Doação de Órgãos e Tecidos para Transplantes (CIHDOTT) do Pio Sodalício das Damas de Caridade de Caxias do Sul - Hospital Nossa Senhora de Pompéia, na cidade de Caxias do Sul, Rio Grande do Sul.

A população foi constituída por 216 indivíduos com diagnóstico de ME, entre os anos de 2013 a 2017, que atenderam aos seguintes critérios de inclusão: ter completado todos os testes clínicos e exame de imagem complementar conforme protocolo de ME, diagnóstico de ME notificado à Central de Transplantes do Estado do Rio Grande do Sul pela instituição coparticipante e residir/ser oriundo dos municípios da área de abrangência da $5^{a}$ Coordenadoria Regional de Saúde.

Com base nos dados coletados por meio dos prontuários eletrônicos de cada indivíduo, estruturou-se uma planilha no programa Excel. Os dados foram analisados através do programa Statistical Package for the Social Science (SPSS), versão 20.0. Para descrição das variáveis quantitativas foram utilizadas as frequências absolutas e relativas. A associação foi verificada pelo teste de Qui-Quadrado de Wald, adotando-se $p<0,05$ como significância estatística. A força de associação entre doação e as variáveis foram testadas por meio do odds ratio $(O R)$ e seus respectivos intervalos de confiança de $95 \%$.

O estudo foi aprovado pelo Comitê de Ética e Pesquisa com Seres Humanos do Centro Universitário - FSG e do Pio Sodalício das Damas de Caridade de Caxias do Sul - Hospital Nossa Senhora de Pompéia, sob o número do certificado de apresentação para apreciação ética (CAAE): 82452418.1.3001.5331 e parecer 2.547.718.

\section{RESULTADOS}

A amostra foi composta por 216 indivíduos com diagnóstico de ME, onde constatou-se que 104 (48,15\%) deles tornaram-se doadores efetivos. Verificou-se a negativa familiar como a principal causa da não doação, correspondendo a $28,24 \%$ dos casos, seguida da contraindicação médica $(15,28 \%)$ e da parada cardíaca (8,33\%), conforme Tabela 1.

Tabela 1 - Potenciais doadores de um hospital da Serra Gaúcha entre os anos de 2013 a 2017 e motivo da não doação de órgãos e tecidos. Caxias do Sul/RS, Brasil, 2018.

\begin{tabular}{lcc}
\hline \multicolumn{1}{c}{ Variáveis } & $\mathbf{n}(\mathbf{2 1 6})$ & Índice \% \\
\hline Paciente foi doador & & \\
Doador & 104 & 48,15 \\
Não Doador & 112 & 51,85 \\
Motivo da não doação & & \\
Negativa familiar & 61 & 28,24 \\
Contra-indicação médica & 33 & 15,28 \\
Parada Cardíaca & 18 & 8,33 \\
\hline
\end{tabular}

Analisando o perfil dos doadores efetivos, 61 (58,65\%) eram do gênero masculino, com idade média de 47,05 $\pm 18,03$ anos, e $43(41,35 \%)$ do gênero feminino, com idade média de $52,36 \pm 14,74$ anos. Os indivíduos, em sua minoria, ou seja, $51(49,04 \%)$ eram provenientes 
da cidade de Caxias do Sul, e, subsequentemente 53 $(50,96 \%)$ oriundos de outras vinte e quatro cidades pertencentes à área de abrangência da $5^{\text {a }}$ Coordenadoria Regional de Saúde. A distribuição das características gerais está apresentada na Tabela 2.

Observou-se significância estatística entre os doadores efetivos e as variáveis: estado civil, ser solteiro $46(44,23 \%)$ ( $p$ 0,032); setor de internação $(67,31 \%)$ ( p 0,009) para unidade de terapia intensiva (UTI); causa da morte encefálica 49 (47,12\%) (p 0,002) para o acidente vascular cerebral e órgãos captados $45(43,27 \%)$ ( $p$ 0,001) foram, em sua maioria, órgãos sólidos com associação das córneas, apresentados na Tabela 2.

Tabela 2 - Perfil sociodemográfico dos doadores elegíveis de um hospital da Serra Gaúcha e relação com as variáveis avaliadas através do teste Qui-Quadrado. Caxias do Sul/RS, Brasil, 2018.

\begin{tabular}{|c|c|c|c|}
\hline Variáveis & n (104) & Índice \% & p-valor \\
\hline \multicolumn{4}{|l|}{ Gênero } \\
\hline Masculino & 61 & 58,65 & 0,839 \\
\hline Feminino & 43 & 41,35 & \\
\hline \multicolumn{4}{|l|}{ Idade média } \\
\hline Masculino & 47,05 & & \\
\hline Feminino & 52,36 & & 0,802 \\
\hline \multicolumn{4}{|l|}{ Cidade } \\
\hline Caxias do Sul & 51 & 49,04 & \\
\hline Outras cidades* & 53 & 50,96 & 0,389 \\
\hline \multicolumn{4}{|l|}{ Religião } \\
\hline Católica & 87 & 83,65 & 0,163 \\
\hline Evangélica & 11 & 10,58 & \\
\hline Outras** & 6 & 5,77 & \\
\hline \multicolumn{4}{|l|}{ Escolaridade } \\
\hline Não alfabetizado & 01 & 0,96 & \\
\hline Ensino fundamental completo & 17 & 16,35 & \\
\hline Ensino fundamental incompleto & 47 & 45,19 & 0,389 \\
\hline Ensino médio completo & 24 & 23,08 & \\
\hline Ensino médio incompleto & 08 & 7,69 & \\
\hline Superior complete & 03 & 2,88 & \\
\hline Superior incomplete & 02 & 1,92 & \\
\hline Pós-graduação & 02 & 1,92 & \\
\hline \multicolumn{4}{|l|}{ Cor } \\
\hline Branca & 84 & 80,77 & 0,052 \\
\hline Parda & 19 & 18,27 & \\
\hline Negra & 01 & 0,96 & \\
\hline
\end{tabular}

Tabela 2 - Continuação

\begin{tabular}{|c|c|c|c|}
\hline Variáveis & n (104) & Índice \% & p-valor \\
\hline \multicolumn{4}{|l|}{ Estado civil } \\
\hline Solteiro & 46 & 44,23 & $0,032 * * *$ \\
\hline Casado & 43 & 41,35 & \\
\hline Outros & 02 & 1,92 & \\
\hline Divorciado & 08 & 7,70 & \\
\hline Viúvo & 03 & 2,88 & \\
\hline União estável & 02 & 1,92 & \\
\hline \multicolumn{4}{|l|}{ Setor de internação } \\
\hline UTI & 70 & 67,31 & $0,009 * * *$ \\
\hline Pronto-Socorro & 25 & 24,04 & \\
\hline Outros hospitais & 08 & 7,69 & \\
\hline SARE & 01 & 0,96 & \\
\hline \multicolumn{4}{|l|}{ Causa da morte encefálica } \\
\hline AVC & 49 & 47,12 & $0,002^{* * *}$ \\
\hline TCE & 35 & 33,65 & \\
\hline Meningites/encefalites & 02 & 1,92 & \\
\hline $\begin{array}{l}\text { Aneurisma/hemorragia } \\
\text { subaracnóidea }\end{array}$ & 13 & 12,51 & \\
\hline Parada Cardíaca & 02 & 1,92 & \\
\hline Hipertensão intracraniana & 03 & 2,88 & \\
\hline \multicolumn{4}{|l|}{$\begin{array}{l}\text { Exame complementar de } \\
\text { imagem }\end{array}$} \\
\hline Angiotomografia & 60 & 57,69 & 0,486 \\
\hline Arteriografia & 41 & 39,42 & \\
\hline Cintilografia & 03 & 2,88 & \\
\hline \multicolumn{4}{|l|}{ Órgãos captados } \\
\hline Sólidos e corneas & 45 & 43,27 & $0,001^{* * *}$ \\
\hline Sólidos, córneas e ossos & 29 & 27,88 & \\
\hline Somente sólidos & 25 & 24,04 & \\
\hline Somente corneas & 3 & 2,88 & \\
\hline Sólidos e ossos & 2 & 1,92 & \\
\hline
\end{tabular}

*Outras 24 cidades da 5a Coordenadoria Regional de Saúde.

**No prontuário eletrônico constava a opção outras.

***Valores significativamente relacionados com a doação de órgãos (teste Qui-Quadrado).

Unidade de terapia intensiva (UTI).

Sala de recuperação (SARE).

Acidente vascular cerebral (AVC).

Traumatismo cranioencefálico (TCE).

Notou-se aumento no número de doadores efetivos entre 2013 a 2017, passando de 14 para 30 doadores, respectivamente, não havendo significância estatística, de acordo com a Tabela 3. 
Tabela 3 - Número de doadores de órgãos de um hospital da Serra Gaúcha entre os anos de 2013 a 2017. Caxias do Sul / RS, Brasil, 2018.

\begin{tabular}{cccc}
\hline Ano & (n) & $\begin{array}{c}\text { Intervalo de } \\
\text { confiança (95\%) }\end{array}$ & odds ratio* \\
\hline 2013 & 14 & $0,922-3,873$ & 1,89 \\
2014 & 22 & $0,451-1,717$ & 0,88 \\
2015 & 20 & $0,476-1,831$ & 0,933 \\
2016 & 18 & $0,391-1,695$ & 0,814 \\
2017 & 30 & $0,462-1,540$ & 0,843 \\
\hline
\end{tabular}

* Teste odds ratio com intervalo de confiança de $95 \%$.

\section{DISCUSSÃO}

Avaliando o perfil sociodemográfico dos doadores, observou-se que o número de indivíduos do gênero masculino foi maior $(58,65 \%)$ quando comparado aos do gênero feminino (41,35\%), corroborando com outros estudos. ${ }^{710-12}$ Estima-se que os resultados apresentados estejam relacionados ao fato de que os homens sejam mais relutantes em assistir sua própria saúde, agravando casos de doenças crônicas, por se envolverem em maior número em acidentes automobilísticos e casos de violência. ${ }^{12-14}$

Em relação à variável faixa etária, a média de idade encontrada foi de 47,5 anos de idade para indivíduos do gênero masculino e 52,36 anos para indivíduos do gênero feminino, assemelhando-se a outros estudos..$^{8,15}$ Esse achado pode estar relacionado ao processo de envelhecimento da população brasileira e à flexibilização de critérios clínicos que favorecem o processo de doação de órgãos e tecidos.

Quanto ao estado civil, predominou o solteiro $(40,74 \%)$, igualmente apresentado em outros estudos. ${ }^{15,16}$ Não foi possível encontrar estudos científicos descritivos que justificassem tais resultados para fins de comparação.

Como fatores que inviabilizam o processo de doação de órgãos ou tecidos, estão as contraindicações absolutas: tumores malignos, sorologia confirmada ou indeterminada para o vírus da imunodeficiência humana (HIV) e vírus linfotrópico da célula humana I e II, infecções sistêmicas por fungos e vírus, choque séptico por bactérias multirresistentes, tuberculose em atividade e doença de Chagas. A partir da constatação, na ausência de contraindicações, os familiares ou representantes do doador elegível são entrevistados para a possibilidade de realizar a doação de órgãos e tecidos. $^{8}$

A retirada e doação de órgãos de indivíduos falecidos só pode ocorrer mediante autorização do cônjuge ou de familiar, com parentesco até segundo grau, maior de 18 anos. ${ }^{16}$ Acredita-se que pessoas sem vínculo de parentesco, ou seja, que não são considerados responsáveis legais quanto à decisão de doação, podem exercer influência sob os responsáveis pela tomada de decisão. ${ }^{17}$

O presente estudo traz a negativa familiar como principal motivador da não doação, respondendo por $28,24 \%$ dos casos. Sabe-se que a negativa familiar é uma das principais causas da não concretização da doação de órgãos e tecidos. ${ }^{8}$ Nos motivos frequentes para a negativa familiar, destacam-se a oposição do próprio paciente quando vivo à doação, apego familiar ao corpo, não aceitação ou compreensão do processo de $\mathrm{ME}$, ansiedade em relação à morte, incapacidade à tomada de decisões frente à notícia da perda do ente querido, receio de mutilação ao corpo, crenças populares e religiosas, bem como a insensibilidade dos profissionais de saúde em relação à abordagem familiar. ${ }^{9}$

O setor de internação hospitalar de maior correlação com a doação de órgãos foi a UTI, com 70 indivíduos (67,31\%). De acordo com pesquisas anteriores, estimase que de $10 \%$ a $20 \%$ dos potenciais doadores evoluem para parada cardíaca antes do processo de doação de órgãos18. No presente estudo, esse valor foi de $8,33 \%$, podendo apontar o preparo e capacitação da equipe da UTI quanto à correta manutenção do paciente com diagnóstico de ME. Vale destacar que o preparo dos profissionais que compõem as equipes de saúde frente ao tema doação de órgão é essencialmente importante e deve ser fortalecido em todas as áreas das instituições de saúde, independente do grau de complexidade no atendimento e da proximidade da doação.

O reconhecimento e a notificação da ME é um dos eventos de maior impacto no cenário atual de saúde, em virtude da grandiosidade que os transplantes proporcionam. Estudo recente evidencia que mesmo que a ME requeira diagnóstico de notificação compulsória comprovada por qualquer estabelecimento de saúde, apenas $62,1 \%$ dos protocolos abertos foram concluídos, deixando em aberto importantes lacunas de informações imprescindíveis para a análise situacional no local avaliado. ${ }^{19}$

Constatou-se que a causa de ME com maior índice na amostra foi devido a AVC em $47,12 \%$ dos casos, e como causa secundária o TCE em $33,65 \%$ dos casos. Tais causas são prevalentes em todos os estudos pesquisados, com valores próximos aos divulgados pelo Registro Brasileiro de Transplantes (RBT) no ano de 2014 , onde o AVC predominou em $48 \%$ dos casos, seguindo do TCE com $38 \% .{ }^{20}$ A pesquisa reforça os dados apresentados pela literatura em relação ao cenário do perfil dos doadores, bem como as causas da ME. Conforme dados dos últimos anos apresentados na Tabela 3, as doações não apresentaram aumento 
significativo, refletindo dessa forma uma dura realidade negativa para com a assistência de saúde, no que tange às doações de órgãos no país.

Em relação aos órgãos captados, $43,27 \%$ dos indivíduos doaram órgãos sólidos com associação de córneas. As captações que envolveram somente órgãos sólidos atingiram índices de 24,04\% e 2,88\% exclusivamente para córneas. Em outro estudo, a associação de órgãos sólidos como coração, rins e fígado somaram $69 \%$ do total e outros $31 \%$ foram relativo às córneas. ${ }^{15}$

Destacam-se como possíveis fatores limitadores do processo de captação de órgãos sólidos a falta de equipes locais capacitadas para retirada de órgãos, as distâncias a serem percorridas pelas equipes externas de captações e as dificuldades logísticas. Infelizmente, muitas vezes, órgãos deixam de ser transplantados devido a entraves no processo de captação, transporte e efetivação dos transplantes de órgãos no Brasil.

\section{CONCLUSÃO}

A taxa de doação de órgãos e tecidos entre os anos de 2013 a 2017 foi de $48,15 \%$ em uma população de 216 pacientes e a negativa familiar destacou-se como principal motivo para a não doação.
São necessárias ações de sensibilização e conscientização referentes à negativa familiar como causa da não doação. Acredita-se que ainda temos um longo caminho em relação a esse processo, visto que ainda há muitas dúvidas e incertezas para a população em geral. Portanto, cabe aos profissionais de saúde o desenvolvimento de habilidades acerca da comunicação, devendo esta ser clara, sincera e efetiva, com aprimoramento das técnicas de acolhimento das famílias desde o momento da internação até a abordagem referente a ME.

Dentre as associações que se correlacionam com o desfecho da doação, destacam-se o estado civil e o AVC como causa da ME. A captação de órgãos sólidos com associação das córneas foi predominante.

Novos estudos com desenhos mais robustos em diferentes centros e com populações maiores são necessários, para que se possa avaliar de forma mais profunda as associações e desfechos.

Por se tratar de uma pesquisa realizada com amostra específica de um hospital da Serra Gaúcha, não se pode depreender que os resultados encontrados sejam generalizáveis. No entanto, esses dados são de grande importância para a região, visto que a instituição é referência em trauma e neurocirurgia para 48 municípios do estado do Rio Grande do Sul.

\section{ABSTRACT}

There is a significant discrepancy between the demand of patients waiting on transplant queues when compared to the amount of transplants performed. Purpose: To measure the donation rate of organs and tissues from patients diagnosed with brain death from 2013 to 2017. Method: A cross-sectional study developed in the city of Caxias do Sul, RS. Data collection was performed in the first half of 2018 , and data were descriptively analyzed by absolute and relative frequencies. The odds ratio and associations were calculated by the Chi-Square test of Wald, considering $p<0.05$ as statistical significance. Results: The donation rate of organs and tissues was of $48.15 \%$ in a population of 216 patients, and the family negative was the main reason for non-donation (28.24\%). Among the associations correlated to the outcome of the donation, civil status and stroke are the main causes for the ME. Conclusion: New studies with more robust designs in different centers and larger populations are necessary, so that associations and outcomes can be more deeply assessed. Awareness and awareness raising actions are necessary when referring to the family denial as cause for non-donation.

Keywords: Brain Death; Organ Transplantation; Tissue and Organ Procurement; Nursing. 


\section{AGRADECIMENTOS}

Agradecemos à Instituição Pio Sodalício das Damas de Caridade de Caxias do Sul - Hospital Nossa Senhora de Pompéia pela autorização concedida à realização da pesquisa. À Comissão Intra-Hospitalar de Doação de Órgãos e Tecidos para Transplantes (CIHDOTT), em especial à Enfermeira Ana Paula Concatto e ao Técnico de Enfermagem Renan Zucolotto da Silva por toda a cordialidade, auxílio e ensinamentos.

\section{REFERÊNCIAS}

1.Lago PM, Piva J, Garcia PC, Troster E, Bousso A, Sarno MO, et al. Morte encefálica: condutas médicas adotadas em sete unidades de tratamento intensivo pediátrico brasileiras. J. Pediatr. 2007;83(2):133-40.

2.Associação Brasileira de Transplante de Órgãos (ABTO). Registro Brasileiro de Transplantes. Dados numéricos da doação de órgãos e transplantes realizados por estado e instituição no período: janeiro / dezembro - 2012. 2012;18(4):22-5.

3.Marconi L, Moreira P, Parada B, Bastos C, Roseiro A, Mota A. Donor cause of brain death in renal transplantation: a predictive factor for graft function? Transplant Proc. 2011;43(1):74-6.

4.Freire SG, Freire ILS, Pinto JTJM, Vasconcelos QLDAQ, Torres GV. Alterações fisiológicas da morte encefálica em potenciais doadores de órgãos e tecidos para transplantes. Esc. Anna Nery. 2012;16(4):761-6.

5.Westphal GA, Garcia VD, Souza RL de, Franke CA, Vieira KD, Birckholz VRZ et al. Diretrizes para avaliação e validação do potencial doador de órgãos em morte encefálica. Rev. bras. ter. Intensiva. 2016;28(3):220-55.

6.Associação Brasileira de Transplantes ABTO. Registro Brasileiro de Transplantes RBT. Dados numéricos da doação de órgãos e transplantes realizados por estado e instituição no período de Janeiro/Junho de 2016. [Internet] [acesso em 2017 Set 12]. Disponível em: http://www.abto.org.br/abtov03/ Upload/file/RBT/2016/1sem-RBT2016\%20leitura.pdf.

7.Associação Brasileira de Transplantes ABTO. Registro Brasileiro de Transplantes RBT. Dados numéricos da doação de órgãos e transplantes realizados por estado e instituição no período de Janeiro/Junho de 2017. [Internet] [acesso em 2017 Set 12]. Disponível em: http://www.abto.org.br/abtov03/ Upload/file/RBT/2017/rbt-leitura-sem.pdf.

8.Noronha MGO, Seter GB, Perini LD, Salles FMO de, Nogara MAS. Estudo do perfil dos doadores elegíveis de órgãos e tecidos e motivos da não doação no Hospital Santa Isabel em Blumenau, SC. Rev AMRIGS. 2012;56(3):199-203.

9.Brandalise M, Pagnussat N. Panorama sobre os transplantes de órgãos no Brasil e fatores associados à baixa adesão da prática. J Bras Transpl. 2015;8(2):50-6.
10. Gois RSS, Galdino MJQ, Pissinati PSC, Pimentel RRS, Carvalho MDB, Haddad MCFL. Efetividade do processo de doação de órgãos para transplantes. Acta Paul Enferm. 2017;30(6):621-7.

11. Freire IL, Silva MF, Gomes AT, Dantas BA, Torres GV. Characterization of the potential donors and structure of hospital units that develops the transplantations. Ciênc Cuid Saúde. 2015;14(3):1281-9.

12. Rodrigues TB, Vasconcelos MI, Brito MC, Sales DS, Silva $\mathrm{RC}$, Souza AM. [Profile of potential organ donors in a reference hospital]. Rev Rene. 2013;14(4):713-9.

13. Couto MT, Pinheiro TF, Valença O, Machin R, Da Silva GSN, Gomes $\mathrm{R}$ et al. Men in primary healthcare: discussing (in) visibility based on gender perspectives. Interface Comun Saúde Educ. 2010;14(33):257-70.

14. Freitas RA, Dell'Agnolo CM, Alves EF, Benguella EA, Pelloso SM, Carvalho MD. Organ and tissue donation for transplantation from fatal trauma victims. Transplant Proc. 2015;47(4):874-8.

15. Bernardes ARB, Almeida CG. Estudo do perfil dos doadores elegíveis de órgãos e tecidos no Hospital de Clínicas da Universidade Federal de Uberlândia / MG. J Bras Transpl. 2015;18(2):34-64.

16. Barreto BS, Santana RJB, Nogueira EC, Fernandes BO, Brito FPG. Fatores relacionados à não doação de órgãos de potenciais doadores no estado de Sergipe, Brasil. Rev. Bras. Pesq. Saúde, Vitória, 2016;18(3):40-8.

17. Pessoa JLE, Schirmer J, Roza BA. Avaliação das causas de recusa familiar a doação de órgãos e tecidos. Acta Paul Enferm. 2013;26(4):323-30.

18. Rossetti AO, Logroscino G, Liaudet L, Ruffieux C, Ribordy V, Schaller MD et al. Status epilepticus: anindependent outcome predictor after cerebral anoxia. Neurology. 2007;69:255-60.

19. Souza BSJ, Lira GG, Mola R. Notificação de morte encefálica em ambiente hospitalar. Rev Rene. 2015;16(2):194-220.

20. Associação Brasileira de Transplantes ABTO. Registro Brasileiro de Transplantes. Dimensionamento dos transplantes no Brasil e em cada estado. 2007-2014, ano XX, 4. [Internet] [acesso em 2017 Out 10]. Disponível em: http://www.abto.org. br/abtov03/Upload/file/RBT/2014/rbt2014-lib.pdf. 